myocardial injury (e.g. echocardiogram evidence of RV strain or raised Troponin) is recognised ("sub-massive" PE) where considerable debate remains as to reliable prognostication and appropriateness of thrombolysis.

Aims Recognising a tendency in our district general hospital to treat PE aggressively, we sought to assess our own practise with regards to thrombolytic therapy in PE to better understand our interpretation of NICE guidance and the wider literature.

Objective Assess routine quantification of haemodynamic instability of the acutely unwell patient in the clinical environment and the extent with which knowledge of right ventricular compromise and cardiac biomarkers influenced decision to thrombolyse.

Methods Retrospective review of case records. Data was extracted from the medical records by one of the authors followed by joint scrutiny by all authors.

Results From June 2010 over 24 months, 17 patients ( 6 males, 11 females) have been thrombolysed. Median age 62 (range 24-90). Of these one patient died 4 days later of sepsis and multi organ failure, and one developed a haematoma in her arm which resolved with conservative management.

Of these patients only two Massive i.e. haemodynamically unstable with one thrombolysed on the ITU. In the submassive PEs, supporting evidence for thrombolysis was CT in 5 cases, and echo 5 , both in 2, and lab (troponin rise) in 9 cases.

Conclusion Thrombolysis in PE especially in submassive PEs remains an area of controversy and clinically a dilemma at times. Our case series shows that patient selection supported by relevant investigations, appropriate patients can benefit without any untoward events.

References

NICE CG144 Venous thromboembolic diseases: full guideline. Jaff MR et al. Management of massive and submassive pulmonary embolism, iliofemoral deep vein thrombosis, and chronic thromboembolic pulmonary hypertension: a scientific statement from the American Heart Association. Circulation 2011.

\section{P145 THROMBOLYSIS OF ACUTE PE PATIENTS REDUCES SUBSEQUENT DEVELOPMENT OF CTEPH}

doi:10.1136/thoraxjnl-2012-202678.428

D C Thomas, R M Limbrey. University Hospital Southampton NHS Foundation Trust, Southampton, United Kingdom

Introduction Pulmonary Embolism (PE) is a frequent diagnosis and the incidence of Chronic Thromboembolic pulmonary hypertension (CTEPH) after a single acute episode of PE is higher than expected - in one prospective long-term study $3.8 \%$ at 2 years (Pengo et al 2004). A diagnosis of CTEPH carries a $30 \% 5$ year mortality and early diagnosis is vital if treatment is to be of success although successful treatment options remain limited.

We hypothesized that by following up all new diagnoses of PE we would pick up early cases of CTEPH and identify risk factors for those developing CTEPH. We also hypothesized that thrombolysis as per the BTS guidelines would reduce the subsequent incidence of CTEPH.

Methods A retrospective study of all patients referred to a PE clinic over a 2 year period was performed. Initial and follow up echocardiograms were examined, provoking factors and treatment identified with a primary end point of development of CTEPH at 2 years. Results Of the first 50 patients presenting with an acute PE $12 \%$ $(n=6)$ had evidence of CTEPH on echocardiogram after 2 years. The major risk factor for the development of CTEPH was an initial echocardiogram demonstrating a RVSP $>50 \mathrm{mmHg}$ which conferred a 5 -fold increase in persistent pulmonary hypertension on echocardiogram at 2 years ( $36 \%$ versus $7 \%$ ).

None of the thrombolysed patients went on to develop CTEPH despite having in $50 \%$ an RVSP $>50 \mathrm{mmHg}$ and all had a normal early (within six months) repeat echocardiogram.
Conclusions Patients who present with an acute PE and have an initial RVSP of $>50 \mathrm{mmHg}$ on echocardiogram have a 5 fold increase in developing persistent pulmonary hypertension at 2 years. However patients who had a RVSP $>50 \mathrm{mmHg}$ at diagnosis and are thrombolysed do not appear to develop CTEPH and have normal echocardiography at 2 years. Bearing in mind the mortality CTEPH carries and the difficulty in treating it, patients presenting with an acute significant $\mathrm{PE}$ and a RVSP $>50 \mathrm{mmHg}$ should be considered for thrombolysis regardless of haemodynamic compromise.

Reference

1. Pengo V, Lensing AWA, Prins $M H$, et al., Incidence of chronic thromboembolic pulmonary hypertension after pulmonary embolism, N Engl J Med, 2004; 350:2257-64.

\section{P146 CAN RAISING THE D-DIMER THRESHOLD SAFELY REDUCE THE NUMBER OF CT PULMONARY ANGIOGRAMS PERFORMED IN SUSPECTED PULMONARY EMBOLISM?}

doi:10.1136/thoraxjnl-2012-202678.429

0 Orhan, C King, A Salam, V Mak. North West London Hospitals NHS Trust, London, England

Introduction CT Pulmonary Angiography (CTPA) is the gold standard investigation for suspected pulmonary embolism (PE). Low or intermediate probability clinical prediction (e.g. Wells score) combined with a negative D-dimer effectively rules out $\mathrm{PE}$ in over $97 \%$ of cases, avoiding the need for CTPA and its inherent risks (radiation exposure and contrast induced nephropathy). This is the recommendation of the BTS guidelines. We undertook a study to examine if the BTS guidelines were being adhered to in our Trust, and whether increasing the D-dimer threshold may safely reduce the need for CTPA.

Methods We obtained a list of CTPAs performed within the Trust between September 2009 and September 2011 and searched our pathology system for a contemporary D-dimer result (HemosIL latex immuno-assay). For all patients with a negative D-dimer ( $\leq 230 \mathrm{ng} /$ $\mathrm{mL}$ ), we looked for a documented pre-test probability score in the clinical notes or calculated a Wells score if not documented. We then analysed CTPA results with D-dimer between 230-500 ng/mL.

Results There were 1645 CTPAs performed during the study period, of which $15 \%$ had confirmed PE $903(54.9 \%)$ had a contemporary D-dimer result, and of these $57(6.3 \%)$ had a negative D-dimer, and $193(21.4 \%)$ were between $230-500 \mathrm{ng} / \mathrm{mL}$. In the negative D-dimer group, 3 (5.3\%) had confirmed PE's on CTPA. One was on tranexamic acid, which can falsely lower D-dimer, and 2 had prolonged admissions in whom $\mathrm{D}$-dimer testing was not appropriate. We were able to examine the notes of $39 / 57$ cases and only $3(8 \%)$ had a pre-test probability documented. On review $31 / 39$ (79.5\%) had a low to intermediate Wells score and should not have had a CTPA.

In the $230-500 \mathrm{ng} / \mathrm{mL} \mathrm{D}$-dimer group, there were only $4 / 193$ (2.1\%) positive CTPAs with a negative predictive value $98 \%$.

Conclusions In our Trust, the lack of pre-test probability scoring combined with $\mathrm{D}$-dimer is leading to inappropriate CTPAs. The rate of $\mathrm{PE}$ in the patients with a $\mathrm{D}$-dimer between $230-500 \mathrm{ng} / \mathrm{mL}$ is also very low. A protocol recommending initial treatment pending an urgent respiratory team review prior to CTPA, could safely reduce the number performed in this group, if combined with a low to intermediate pre-test probability score.

\section{P147 THE WELLS PE SCORE - AN EFFECTIVE PRE-TEST PROBABILITY TOOL?}

doi:10.1136/thoraxjnl-2012-202678.430

J Karalis, J Curtin Norfolk. orwich University Hospital, Norwich, United Kingdom

Introduction and Objectives British Thoracic Society guidelines advise all patients with possible PE should have pre-test clinical 
probability assessed and documented. The Norfolk and Norwich University Hospital (NNUH) uses the Wells PE score. Validation studies have shown it to be a sensitive tool with a positive predictive value of: low risk (5\%), medium risk (25\%), and high risk (70\%) respectively.

We wanted to assess the correlation of the Wells PE score with PE positivity rates on CT Pulmonary Angiogram (CTPA) in our patient population. We anticipated similar positive predictive rates as those seen in the original validation studies.

Methods We retrospectively collected data from CTPA requests submitted between October 2009 to September 2010. The Wells PE score was used to risk stratify patients into low (score $<2$ ), moderate (score 2-6), or high risk (score $>6$ ). These were correlated with the outcome of the CTPAs. We also used the alternate dichotomised Wells PE score of PE Unlikely (score $\leq 4)$ and PE likely group (score $>4$ ) and correlated with results.

Results 1160 CTPA requests were identified, 66 were excluded, (they lacked a wells PE score). 1094 CTPA requests were analysed.

Conclusion We found poor correlation between the pre-test probability score and confirmed PE;

Results show a positive predictive value of low risk (21.4\%), medium risk (22.1\%) and high risk (35.3\%). The dichotomised score showed PE Unlikely (19.1\%) and PE Likely (27.4\%). Analysis of the Wells PE variables revealed that they lacked a high specificity and high sensitivity, the subjective variable demonstrated the lowest specificity.

The Wells PE score is not an effective pre-test probability tool for discriminating low, moderate and high risk groups in the patient population at $\mathrm{NNUH}$

\section{Reference}

1. Wells PS, Anderson DR, Rodger M, et al. Derivation of a simple clinical model to categorise patients probability of pulmonary embolism: Increasing the models utility with the SimpliRED D-dimer. Thrombosis and Haemastasis. 2000; 83 (3): 416-420

Abstract P147 Table 1

\begin{tabular}{lcccc}
\hline & CTPAs & + ve for PE & -ve for PE & Positivity rate \\
\hline Total & 1094 & 278 & 816 & $25.4 \%$ \\
Low risk & 56 & 12 & 44 & $21.4 \%$ \\
Medium risk & 763 & 169 & 594 & $22.1 \%$ \\
High risk & 275 & 97 & 178 & $35.3 \%$ \\
$<4$ PE unlikely & 261 & 50 & 211 & $19.1 \%$ \\
$>4$ PE likely & 833 & 228 & 605 & $27.4 \%$
\end{tabular}

\section{P148 THE LIES WE TELL - PRE-TEST PROBABILITY IS ONLY USEFUL AT RISK STRATIFYING PULMONARY EMBOLI WHEN USED ACCURATELY}

doi:10.1136/thoraxjnl-2012-202678.431

M Newnham, H Stone, S Salehi-Bird, R Summerfield, N Mustfa. University Hospital of North Staffordshire, Stoke-on-Trent, United Kingdom

Introduction Pre-test probability (PTP) scores are widely used to risk stratify pulmonary emboli (PE). A multitude of scores exist; however diagnosing PE remains challenging. We aim to identify whether patients were correctly PTP scored and what effect rescoring with the correct information has on predicting PE. We will identify the effect different PTP scores have on the likelihood of PE.

Methods We conducted a retrospective study of inpatient computer tomography pulmonary angiograms (CTPA) within a large teaching hospital over a 1-year period. We recorded the originally submitted PTP score (modified Wells score), D-Dimer result and the CTPA outcome (PE-positive or negative cohorts). The accuracy of the PTP score was assessed from the original clinical information; rescoring when appropriate. We also rescored using alternative PTP systems (Geneva and simplified BTS). We analysed whether any factor or PTP scores could predict the presence of PE.

Results 202 CTPAs were performed (70, 35\% PE positive; 131, 65\% PE negative). Baseline characteristics did not differ (age, gender, requesting team, inflammatory markers, mortality). $\mathrm{PE}$ was more likely with a higher D-dimer (682 vs. 853; p<0.001); pneumonia occurred more frequently in the PE-negative cohort (61 vs. 12; $p<0.001$ ). A third of forms were scored incorrectly; occurring significantly more in the PE-negative cohort $(p=0.001)$. There was no difference between the original PTP scores (3.7 vs. 3.8; $p=0.631$ ); however when rescored with the correct clinical information, the PE-positive cohort had a significantly higher PTP score (2.0 vs. $3.1 ; p=0.001)$ (Table 1). Geneva score did not accurately predict PE (2.3 vs. 2.5; $p=0.25)$; whereas the simplified BTS score did (0.9 vs. 1.3; $p<0.001)$.

Conclusions Patients without a PE were significantly more likely to have an incorrect PTP score. Clinicians may have poor concordance with scoring to meet CTPA requesting criteria. The originally calculated PTP score was not predictive of PE. However, when scores were adjusted with the correct information, a higher PTP score was predictive of PE. Simple PTP scoring systems performed well compared with more complex versions. PTP scores are only effective at predicting PE when used accurately; this may not occur in practise.

Abstract P148 Table 1 PTP scores in negative and positive PE cohorts and the statistical significance between them

\begin{tabular}{lccc}
\hline PTP Score & PE -ve & PE $+\mathbf{v e}$ & p-value \\
\hline Wells - original & 3.7 & 3.8 & 0.631 \\
Wells - rescore & 2.0 & 3.1 & 0.001 \\
Geneva & 2.3 & 2.5 & 0.250 \\
Simplified BTS & 0.9 & 1.3 & $<0.001$ \\
\hline
\end{tabular}

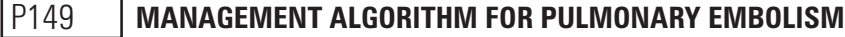
(PE) WITH RIGHT HEART STRAIN: A CASE SERIES

doi:10.1136/thoraxjnl-2012-202678.432

V Smith, G Tunnicliffe, G Fiorino, A Draper, I Vlahos, M Shannon, BP Madden. St George's Hospital, London, UK

Current guidelines state thrombolysis is first line therapy in hypotensive PE patients and may be of benefit in normotensive patients with right heart strain. There is, however, no specific guidance on selecting these patients.

In 2010, St George's Hospital, under the guidance of a multidisciplinary team of specialists, initiated an algorithm for the management of patients with massive PE. The aim of this study was to determine whether the algorithm is an effective means of assessing a series of nine patients who received thrombolytic therapy over a two year period

The age range of the patients was 36-81 years, 5 were male.5 patients had identifiable thromboembolic risk factors: pregnancy and protein $S$ deficiency (1), recent lower limb surgery (2), new cancer diagnosis (1) and chronic immobility (1). All 9 patients had a computed tomography pulmonary angiogram (CTPA) confirming large proximal PEs with elevated troponin I and NT-pro-BNP levels at diagnosis. Right heart strain was demonstrated on echocardiogram in five patients with evidence of intracardiac thrombus in two. The remaining patients had right heart strain demonstrated by CTPA. The indications for thrombolysis were cardiac arrest (1), hypotension (1), intracardiac thrombus (2) and significant right ventricular strain(5).There was one mortality within this cohort following retroperitoneal bleed. This patient underwent an echocardiogram 5 days post thrombolysis revealing severely dilated right heart and RVSP of $61 \mathrm{mmHg}$. Patients achieved good resolution of thrombus on repeat CTPA, and no evidence of right heart strain on follow up echocardiogram. 